Revista Brasileira de Agricultura Irrigada v.10, nº.4, p. 830 - 840, 2016

ISSN 1982-7679 (On-line)

Fortaleza, CE, INOVAGRI - http://www.inovagri.org.br

DOI: $10.7127 /$ rbai.v10n400454

Protocolo 454.16 - 23/06/2016 Aprovado em 21/07/2016

\title{
ESTIMATIVA DA EVAPOTRANSPIRAÇÃO DE REFERÊNCIA POR PENMAN- MONTEITH FAO 56 USANDO DADOS METEOROLÓGICOS LIMITADOS EM ARACAJU, SERGIPE
}

\author{
Mairton Gomes da Silva ${ }^{1}$, Lucas dos Santos Batista ${ }^{2}$, Raimundo Rodrigues Gomes Filho ${ }^{3}$, Clayton \\ Moura de Carvalho ${ }^{4}$
}

\section{RESUMO}

O método de Penman-Monteith (PM-FAO 56) é recomendado como padrão para a estimativa da evapotranspiração de referência $\left(\mathrm{ET}_{0}\right)$. Porém, este método requer um grande número de dados de entrada como radiação solar, temperatura do ar, velocidade do vento e umidade relativa, o que tem limitado sua aplicação em muitos locais. Baseado nisto, com o presente trabalho objetivou-se avaliar a estimativa da ETo PM-FAO 56 com dados meteorológicos limitados em Aracaju no Estado de Sergipe, Brasil. O trabalho foi realizado a partir de dados diários de temperatura máxima e mínima do ar, umidade relativa do ar, velocidade do vento e insolação. Foram avaliados três cenários de estimativa de $\mathrm{ET}_{\mathrm{o}}$ PM-FAO 56 com dados limitados: ausência de radiação solar, umidade relativa do ar e velocidade do vento; ausência de radiação solar e velocidade do vento; e ausência de umidade relativa do ar e velocidade do vento. Os valores de $\mathrm{ET}_{\mathrm{o}}$ estimados por PMFAO 56 com dados completos e dados ausentes foram comparados considerado o coeficiente de determinação $\left(\mathrm{R}^{2}\right)$, erro médio de estimativa (MBE) e a raiz do erro do quadrático médio (RMSE). As melhores estimativas de $\mathrm{ET}_{0} \mathrm{PM}-\mathrm{FAO} 56$ foram quando a radiação solar estava disponível, com dados ausentes de umidade relativa do ar (estimada a partir da temperatura mínima) e valores constantes de velocidade do vento entre 1 e $3 \mathrm{~m} \mathrm{~s}^{-1}$, porém quando apenas dados de temperaturas máxima e mínima do ar estão disponíveis não é recomendado estimar a ETo PM-FAO 56 nas condições climáticas locais.

Palavras-chave: Temperatura do ar, radiação solar global, velocidade do vento.

\section{ESTIMATION OF REFERENCE EVAPOTRANSPIRATION BY FAO-56 PENMAN- MONTEITH USING LIMITED WEATHER DATA IN ARACAJU, SERGIPE, BRAZIL}

\footnotetext{
${ }^{1}$ Tecnólogo em Irrigação e Drenagem, Doutorando em Engenharia Agrícola na Universidade Federal do Recôncavo da Bahia, Cruz das Almas, Bahia, Brasil, e-mail: mairtong@hotmail.com

${ }^{2}$ Eng. Agrônomo, Mestrando em Recursos Hídricos na Universidade Federal de Sergipe, Aracaju, Sergipe, Brasil, email: lucasbaptistaufrb@gmail.com

${ }^{3}$ Eng. Agrônomo, Professor adjunto do curso de Engenharia Agrícola na Universidade Federal de Sergipe, e-mail: rrgomesfilho@hotmail.com

${ }^{4}$ Tecnólogo em Recursos Hídricos e Irrigação, Doutor em Engenharia Agrícola pela Universidade Federal do Ceará, Fortaleza, Ceará, Brasil, e-mail: carvalho_cmc@yahoo.com.br
} 


\begin{abstract}
The method Penman-Monteith (FAO-56 PM) is recommended as standard for estimation of reference evapotranspiration $\left(\mathrm{ET}_{\mathrm{o}}\right)$. However, this method requires a large number of input data such as solar radiation, air temperature, relative humidity and wind speed, which has limited its use in many locations. Based on this, the objective of this work was to evaluate the estimation of $\mathrm{ET}_{\mathrm{o}}$ FAO-56 PM using limited weather data in Aracaju, Sergipe State, Brazil. The study was conducted from daily data of maximum and minimum air temperatures, relative humidity, wind speed and

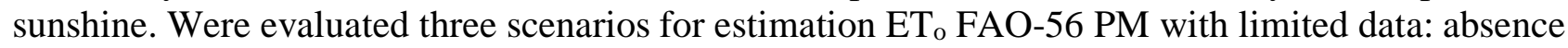
of solar radiation, relative humidity and wind speed; absence of solar radiation and wind speed and absence of relative humidity and wind speed. The values of ETo estimated by FAO-56 PM with complete data and missing data were compared considered the coefficient of determination $\left(\mathrm{R}^{2}\right)$, MBE (mean bias error) and RMSE (root mean square error). The best estimates of ET FAO-56 PM was when solar radiation was available, with missing data of relative humidity (estimated from the minimum temperature) and constant values of wind speed between 1 and $3 \mathrm{~m} \mathrm{~s}^{-1}$, however only when air temperatures are available is not recommended for estimation $\mathrm{ET}_{\mathrm{o}}$ FAO PM on local weather conditions.
\end{abstract}

Keywords: Air temperature, solar radiation, wind speed.

\section{INTRODUÇÃO}

Em regiões semiáridas onde os recursos hídricos são limitados e seriamente ameaçados pela exploração excessiva, é essencial estimar as necessidades hídricas das culturas com maior precisão, exigindo que o planejamento e a gestão da sua utilização ocorram em termos racionais e otimizados (JABLOUN; SAHLI, 2008; SILVA et al., 2015).

Segundo Sentelhas et al. (2010), a evapotranspiração é um parâmetro importante para os estudos climatológicos e hidrológicos, bem como para o planejamento e manejo de irrigação. A evapotranspiração de referência $\left(\mathrm{ET}_{0}\right)$ expressa a demanda evaporativa da atmosfera independente do tipo de cultura, desenvolvimento da cultura e práticas de manejo. Sendo as variáveis meteorológicas os fatores que mais afetam a $\mathrm{ET}_{\mathrm{o}}$. Consequentemente, $\mathrm{ET}_{\mathrm{o}}$ é um parâmetro climático e pode ser calculado a partir de dados meteorológicos. Existem vários métodos para estimar a $\mathrm{ET}_{\mathrm{o}}$, mas suas performances em diferentes condições climáticas podem variar, uma vez que eles têm algum empirismo em sua concepção.

Buscando uma padronização nas estimativas de $\mathrm{ET}_{\mathrm{o}}$, a Organização das Nações
Unidas para Agricultura e Alimentação (FAO) e a Comissão Internacional de Irrigação e Drenagem (ICID) em seu boletim 56 adotou como padrão o método de PenmanMonteith (PM-FAO 56) (ALLEN et al., 1998).

A principal complicação na estimativa de $E T_{0}$ usando esse modelo é a exigência de dados meteorológicos que podem não ser facilmente disponíveis, restringindo seu uso em vários locais (MOHAWESH, 2011). Nesse sentido, para solucionar tal problema, a FAO recomenda estimar a $\mathrm{ET}_{\mathrm{o}}$ a partir da importação de dados de uma estação que está sob as mesmas condições climáticas ou a partir de dados de temperatura máxima e mínima do ar (ALLEN et al., 1998). Neste último procedimento, a radiação solar é estimada com dados de temperatura máxima e mínima, a umidade relativa obtida em função da pressão parcial de vapor d’água (estimada a partir da temperatura mínima) e recomendase utilizar o valor médio de velocidade do vento de $2 \mathrm{~m} \mathrm{~s}^{-1}$.

Tais procedimentos têm exigido sua avaliação em diferentes países e climas para testar sua viabilidade, como feito na Sérvia (TRAJKOVIC, 2005; TRAJKOVIC; KOLAKOVIC, 2009; TRAJKOVIC et al., 2011); no sul da Bulgária (POPOVA et al., 
2006); na Tunísia (JABLOUN; SAHLI, 2008); e Austral (WANG et al., 2011); na Turquia (BENLI et al., 2010); nos Estados Unidos da América (GOCIC; TRAJKOVIC, 2010; MARTINEZ; THEPADIA, 2010; ROJAS; SHEFFIELD, 2013); no sul de Ontário no Canadá (SENTELHAS et al., 2010); no Irã (MAJIDI et al., 2015); em países do Mediterrâneo (LÓPEZ-MORENO et al., 2009) e no Equador (CÓRDOVA et al., 2015).

No Brasil este pocedimento já foi tEstado em diferentes regiões, no Nordeste (SILVA et al., 2010a; SILVA et al., 2010b; ROCHA et al., 2011; SILVA et al., 2013; MORAIS et al., 2015); no Sudeste (CARVALHO et al., 2013; CARVALHO et al., 2015; ALENCAR et al., 2015) e no Sul (MINUZZI et al., 2014).

Diante do exposto, o objetivo deste trabalho foi avaliar a estimativa da evapotranspiração de referência $\left(\mathrm{ET}_{0}\right)$ a partir de dados meteorológicos limitados, em Aracaju no Estado de Sergipe, Brasil.

\section{MATERIAL E MÉTODOS}

O presente trabalho foi realizado a partir de dados médios diários de temperatura máxima e mínima do ar, umidade relativa do ar, velocidade do vento e insolação, oriundos da estação meteorológica convencional de Aracaju, Sergipe $\left(10,95^{\circ}\right.$ Sul, $37,04^{\circ}$ Oeste e altitude de $4,72 \mathrm{~m}$ acima do nível mar), compreendendo o período de 2000 a 2015. Os dados foram obtidos junto à rede de estações do Instituto Nacional de Meteorologia (INMET), a partir Banco de Dados Meteorológicos para Ensino e Pesquisa (BDMEP).

A evapotranspiração de referência $\left(\mathrm{ET}_{0}\right)$ diária foi estimada pelo método padrão Penman-Monteith com dados completos e mínimos (Equação 1), com auxílio de planilhas eletrônicas. Os procedimentos de estimativa dos parâmetros para cálculo da $\mathrm{ET}_{\mathrm{o}}$ contando apenas com dados de temperatura máxima e mínima do ar segue a metodologia sugerida pelo boletim número 56 da $\mathrm{FAO}$ (ALLEN et al., 1998).

$$
\mathrm{ET}_{\mathrm{o}} \mathrm{PM}=\frac{0,408 \Delta\left(\mathrm{R}_{\mathrm{n}}-\mathrm{G}\right)+\gamma \frac{900}{\mathrm{~T}_{\text {méd }}+273} \mathrm{u}_{2}\left(\mathrm{e}_{\mathrm{s}}-\mathrm{e}_{\mathrm{a}}\right)}{\Delta+\gamma\left(1+0,34 \cdot \mathrm{u}_{2}\right)}
$$

em que: $\mathrm{ET}_{0} \mathrm{PM}$ é a evapotranspiração de referência por Penman-Monteith, em mm dia ${ }^{-}$ ${ }^{1} ; \mathrm{R}_{\mathrm{n}}$ é a radiação líquida total, em $\mathrm{MJ} \mathrm{m}^{-2}$ dia $^{-}$ 1; G é a densidade do fluxo de calor no solo, em $\mathrm{MJ} \mathrm{m}^{-2} \mathrm{dia}^{-1}$; $\mathrm{T}_{\text {méd }}$ é a temperatura média diária do ar, em ${ }^{\circ} \mathrm{C}$; $\mathrm{u}_{2}$ é velocidade do vento média diária a $2 \mathrm{~m}$ de altura, em $\mathrm{m} \mathrm{s}^{-1}$; $\mathrm{e}_{\mathrm{s}}$ é a pressão de saturação de vapor, em $\mathrm{kPa}$; $\mathrm{e}_{\mathrm{a}}$ é a pressão parcial de vapor, em $\mathrm{kPa}$; $\mathrm{e}_{\mathrm{s}}-\mathrm{e}_{\mathrm{a}}$ é o déficit de saturação de vapor, em $\mathrm{kPa} ; \Delta$ é a declividade da curva de pressão de vapor no ponto de $\mathrm{T}_{\text {méd, }}$ em $\mathrm{kPa}{ }^{\circ} \mathrm{C}^{-1} ; \gamma$ é o coeficiente psicrométrico, em $\mathrm{kPa}^{\circ} \mathrm{C}^{-1}$.

Foram avaliados três cenários para estimativa de $\mathrm{ET}_{\mathrm{o}} \mathrm{PM}-\mathrm{FAO} 56 \mathrm{com}$ dados meteorológicos em falta: dados ausentes de radiação solar, umidade relativa do ar e velocidade do vento $\left(\mathrm{u}_{2}\right)$; dados ausentes de radiação solar e velocidade do vento; e dados ausentes de umidade relativa do ar e velocidade do vento. Para os cenários com dados de $\mathrm{u}_{2}$ em falta, assumiram-se cinco cenários diferentes, cada um representado por um valor constante de $\mathrm{u}_{2}$, quais sejam: $1,2,3$, 4 e $5 \mathrm{~m} \mathrm{~s}^{-1}$, respectivamente.

A estimativa da radiação solar global foi baseada na diferença entre as temperaturas máxima e mínima do ar e o fator associado à posição do local da estação (Equação 2).

$$
\mathrm{R}_{\mathrm{s}}=\mathrm{K}_{\mathrm{rs}} \sqrt{\left(\mathrm{T}_{\text {máx }}-\mathrm{T}_{\text {mín }}\right)} \cdot \mathrm{R}_{\mathrm{a}}
$$

em que: $R_{s}$ e $R_{a}$ são a radiação solar e no topo da atmosfera, respectivamente, em $\mathrm{MJ} \mathrm{m}^{-2}$ dia $^{-1} ; T_{\text {máx }}$ e $T_{\text {mín são as temperaturas máxima }}$ e mínima do ar, em ${ }^{\circ} \mathrm{C}$; $\mathrm{K}_{\mathrm{rs}}$ é o coeficiente de 
ajuste, adimensional. $\mathrm{O} \mathrm{K}_{\mathrm{rs}}$ é empírico e difere para regiões do interior e litoral: para o interior, onde a massa de terra domina e as massas de ar não são influenciadas por uma grande massa de água, $\mathrm{K}_{\mathrm{rs}}$ é de 0,16; para locais situados no litoral ou adjacente à costa de uma massa de terra grande e onde as massas de ar são influenciadas por um corpo de água, $\mathrm{K}_{\mathrm{rs}}$ é de 0,19 (ALLEN et al., 1998). O valor de $\mathrm{K}_{\mathrm{rs}}$ utilizado no presente trabalho foi de 0,19 .

A radiação solar no topo da atmosfera foi obtida conforme os procedimentos sugeridos por Allen et al. (1998) (Equações $\quad 3 \quad$ a $\quad$ 6).

$$
\begin{array}{r}
R_{a}=\frac{24 \times 60}{\pi} G_{s c} \cdot d_{r} \\
d_{r}=1+0,033 \cdot \cos \cdot\left(\frac{2 \cdot \pi \cdot J}{365}\right) \\
\omega_{s}=\cos ^{-1}(-\tan \varphi \cdot \tan \delta) \\
\delta=0,409 \cdot \operatorname{sen}\left(\frac{2 \cdot \pi \cdot J}{365}-1,39\right)
\end{array}
$$

em que: $\mathrm{G}_{\mathrm{sc}}$ é uma constante, 0,0820 $\mathrm{MJ} \mathrm{m}^{-2}$ $\min ^{-1} ; \mathrm{d}_{\mathrm{r}}$ é a distância relativa Terra-Sol; $\delta$ é a declinação solar, radianos; $\varphi$ é a latitude local, radianos; $\omega_{s}$ é o ângulo de radiação no momento do por do sol, radianos; J é o dia Juliano.

O cálculo da umidade relativa do ar foi em função da pressão de saturação de vapor d'água $\left(\mathrm{e}_{\mathrm{s}}\right)$ estimada com base nas temperaturas máxima e mínima do ar, e a pressão parcial de vapor d’água $\left(\mathrm{e}_{\mathrm{a}}\right)$ obtida assumindo a temperatura mínima do ar. De acordo com Allen et al. (1998), a temperatura do ponto de orvalho $\left(\mathrm{T}_{\mathrm{po}}\right)$ pode ser substituída pela temperatura mínima do ar $\left(\mathrm{T}_{\text {mín }}\right)$ $\left(\mathrm{T}_{\mathrm{po}} \approx \mathrm{T}_{\text {mín }}\right)$. Esta afirmação pressupõe implicitamente que, ao amanhecer, quando a temperatura está próxima da $T_{\text {mín, }}$ o ar está praticamente saturado, isto é, a umidade relativa do ar é quase 100\% (Equação 7).

$$
e_{a}=0,6108 \cdot \exp \left(\frac{17,27 \times T_{\min }}{T_{\text {min }}+237,3}\right)
$$

Para o cenário em que os dados ausentes foram umidade relativa do ar e velocidade do vento, a radiação solar global foi calculada em função do número de horas de insolação (ALLEN et al., 1998) (Equação 8).

$$
\mathrm{R}_{\mathrm{s}}=\left(\mathrm{a}+\mathrm{b} \frac{\mathrm{n}}{\mathrm{N}}\right) \cdot \mathrm{R}_{\mathrm{a}}
$$

em que: a e b são coeficientes locais, a $=0,25$ e $\mathrm{b}=0,50 ; \mathrm{n}$ é o número de horas de brilho solar do dia, em h; $\mathrm{N}$ é a duração máxima de brilho solar em um dia, em h (Equação 9).

$$
\mathrm{N}=\frac{24}{\pi} \cdot \omega_{s}
$$

O desempenho da $\mathrm{ET}_{\mathrm{o}}$ estimada por PM-FAO 56 com dados meteorológicos completos e em falta foi avaliado utilizando os parâmetros estatísticos: coeficiente de determinação $\left(\mathrm{R}^{2}\right)$, erro médio de estimativa (MBE) (Equação 10) e a raiz do erro quadrático médio (RMSE) (Equação 11).

$$
\begin{aligned}
M B E & =\frac{1}{\mathrm{n}} \sum_{\mathrm{i}=1}^{\mathrm{n}}\left(\mathrm{ET}_{\mathrm{o}} \mathrm{PM}_{\text {mínimo }}-\mathrm{ET}_{\mathrm{o}} \mathrm{PM}_{\text {completo }}\right) \\
R M S E & =\sqrt{\frac{1}{\mathrm{n}} \sum_{\mathrm{i}=1}^{\mathrm{n}}\left(\mathrm{ET}_{\mathrm{o}} \mathrm{PM}_{\text {mínimo }}-\mathrm{ET}_{\mathrm{o}} \mathrm{PM}_{\text {completo }}\right)^{2}}
\end{aligned}
$$


em que: $\mathrm{ET}_{0} \mathrm{PM}_{\text {mínimo é a evapotranspiração }}$ de referência estimada por PenmanMonteith com dados mínimos, em $\mathrm{mm} \mathrm{dia}^{-1} ; \mathrm{ET}_{0} \mathrm{PM}_{\text {completo é a evapotranspiração }}$ de referência estimada por Penman-Monteith com dados completos, em $\mathrm{mm} \mathrm{dia}^{-1} ; \mathrm{n}$ é o número de observações.

\section{RESULTADOS E DISCUSSÃO}

$\mathrm{Na}$ Figura 1 encontra-se a relação entre as estimativas da $\mathrm{ET}_{0}$ PM-FAO 56 com dados completos e mínimos, com a radiação solar global $\left(\mathrm{R}_{\mathrm{s}}\right)$ estimada a partir das temperaturas máxima e mínima do ar, umidade relativa (UR) obtida em função da pressão parcial de vapor estimada a partir da temperatura mínima do ar e valores constantes de velocidade do vento $\left(\mathrm{u}_{2}\right)$ de $1,2,3$, $\begin{array}{llllll}4 & \text { e } & 5 & \mathrm{~m}^{-1} & \mathrm{~s}^{-1} & \text { respectivamente. }\end{array}$
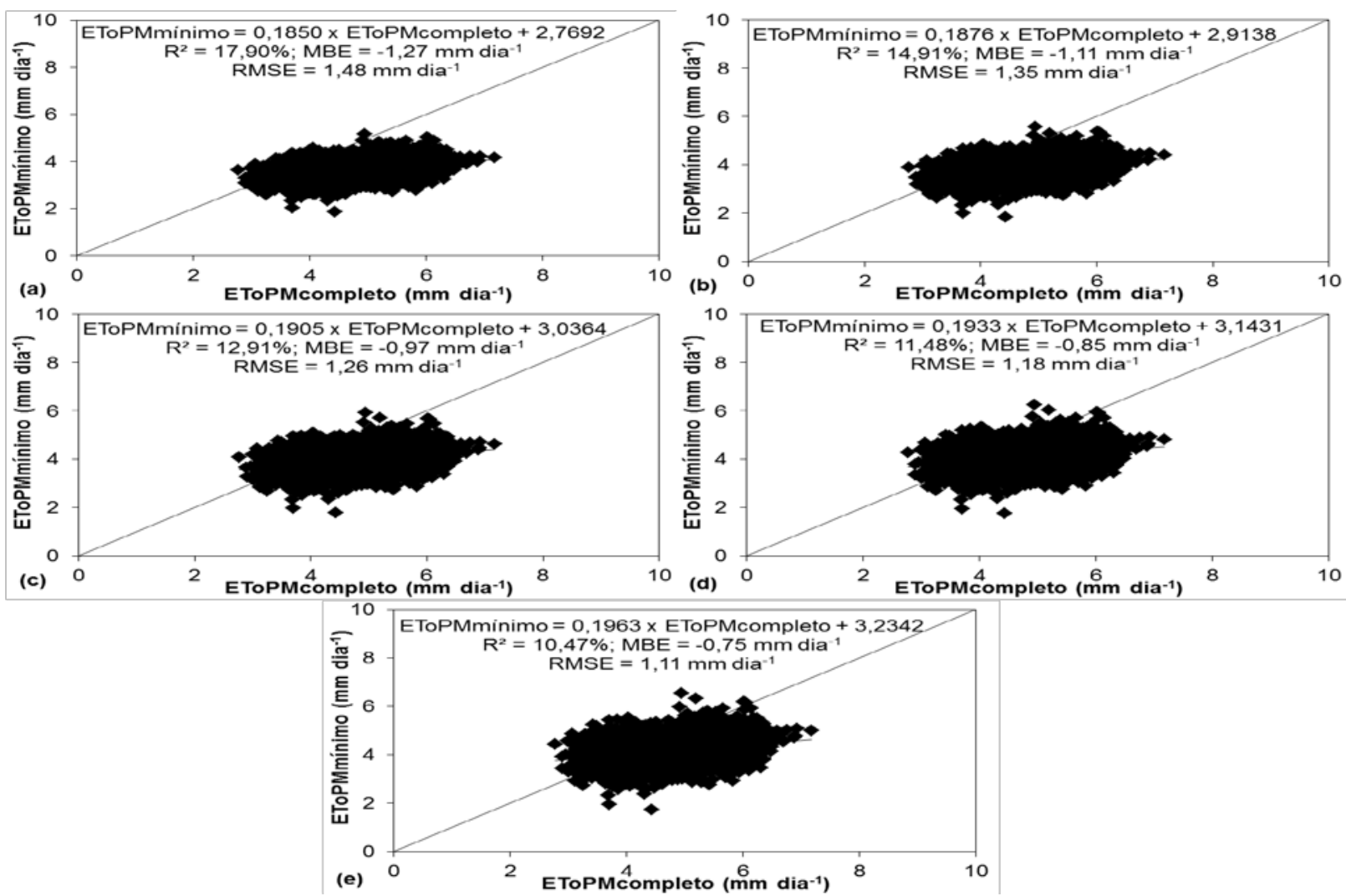

Figura 1. Comparação entre a $E_{0}$ PM-FAO 56 com dados completos e $E_{0}$ PM-FAO 56 com dados mínimos (radiação solar global e umidade relativa do ar estimadas a partir das temperaturas máxima e mínima do ar) e valores fixos de velocidade do vento de $1 \mathrm{~m} \mathrm{~s}^{-1}$ (a), $2 \mathrm{~m} \mathrm{~s}^{-1}$ (b), $3 \mathrm{~m} \mathrm{~s}^{-1}$ (c), $4 \mathrm{~m} \mathrm{~s}^{-1}$ (d) e $5 \mathrm{~m}$ $\mathrm{s}^{-1}(\mathrm{e})$.

Observam-se baixos valores do coeficiente de determinação $\left(\mathrm{R}^{2}\right)$, sendo estes valores reduzidos ainda mais à medida que se aumentou a $\mathrm{u}_{2}$, variando de $17,90 \%$ (Figura 1a) quando foi utilizada a $\mathrm{u}_{2}$ constante de $1 \mathrm{~m}$ $\mathrm{s}^{-1}$ a $10,47 \%$ (Figura 1 e) ao se utilizar a $\mathrm{u}_{2}$ de $5 \mathrm{~m} \mathrm{~s}^{-1}$.
Os baixos valores de $\mathrm{R}^{2}$ refletem em maiores dispersões dos dados, como se pode verificar no indicador $\mathrm{MBE}$, em que a $\mathrm{ET}_{\mathrm{o}}$ PM-FAO 56 com dados mínimos tendeu a subestimar a $\mathrm{ET}_{0}$ PM-FAO 56 com dados completos. Estas subestimativas foram maiores quando se utilizou os menores 
valores constantes de $\mathrm{u}_{2}$, com valores médios de $\mathrm{MBE}$ da ordem de -1,27; -1,11; -0,97; 0,85 e $-0,75 \mathrm{~mm} \mathrm{dia}^{-1}$ para os valores de $\mathrm{u}_{2}$ de $1 \mathrm{~m} \mathrm{~s}^{-1}$ (Figura 1a), $2 \mathrm{~m} \mathrm{~s}^{-1}$ (Figura 1b), $3 \mathrm{~m} \mathrm{~s}^{-}$ 1 (Figura 1c), $4 \mathrm{~m} \mathrm{~s}^{-1}$ (Figura 1d) e $5 \mathrm{~m} \mathrm{~s}^{-1}$ (Figura 1e), respectivamente.

Ainda de acordo com os indicadores estatísticos apresentados na Figura 1, os valores de RMSE variaram de $1,11 \mathrm{~mm} \mathrm{dia}^{-1}\left(\mathrm{u}_{2}=5 \mathrm{~m} \mathrm{~s}^{-1}\right)$ a $1,48 \mathrm{~mm} \mathrm{dia}^{-1}$ $\left(\mathrm{u}_{2}=1 \mathrm{~m} \mathrm{~s}^{-1}\right)$ e, segundo Sentelhas et al. (2010) valores de erros dessa magnitude são muito elevados para estimativas diárias de $\mathrm{ET}_{\mathrm{o}}$. Isto implica que, nas condições climáticas de Aracaju, no Estado de Sergipe, o procedimento de estimar a radiação solar e umidade relativa do ar quando estão disponíveis apenas dados de temperaturas máxima e mínima do ar não é indicado para estimar a ETo PM-FAO 56, devendo o pesquisador e/ou técnico local recorrer a métodos que requeiram o menor número de variáveis de entrada, desde que, a escolha de tais métodos seja validada com o uso de lisímetro.

Os resultados encontrados no presente trabalho estão de acordo com relatos anteriores. De acordo com Benli et al. (2010), no clima semiárido de Ancara na Turquia é preferível quando se tem apenas dados de temperatura do ar utilizar métodos mais simples do que estimar a $\mathrm{ET}_{0} \mathrm{PM}-\mathrm{FAO}$ quando dados de umidade relativa do ar, radiação solar e velocidade do vento estão em falta. Em diferentes localidades na Flórida, Martinez e Thepadia (2010) reportaram superestimativa da $\mathrm{ET}_{0} \mathrm{PM}-\mathrm{FAO}$ com dados mínimos em relação as estimativas com dados completos. Em estudos realizados na Sérvia por Trajkovic (2005), Trajkovic e Kolakovic (2009) e Trajkovic et al. (2011) quando foi utilizado o valor de $\mathrm{u}_{2}$ constante de $2,0 \mathrm{~m} \mathrm{~s}^{-1}$ recomendado pela FAO (ALLEN et al., 1998), os autores alertam que este valor deve ser usado com precaução, especialmente quando dados de radiação solar e/ou umidade relativa não estão disponíveis.
Corroborando com os resultados obtidos em trabalhos realizados na Sérvia, nas condições climáticas de clima semiúmido do Irã, de acordo com Majidi et al. (2015) é recomendável usar $\mathrm{o}$ valor de $\mathrm{u}_{2}$ médio histórico de cada estação ou um valor médio de toda a área de estudo como uma alternativa para a falta de dados de $\mathrm{u}_{2}$. Em estudo de Popova et al. (2006) na Bulgária, quatro procedimentos foram utilizados quando os dados de u2 não estavam disponíveis: importar dados de uma estação próxima; utilizar uma média anual regional para todas as estações $\left(1,68 \mathrm{~m} \mathrm{~s}^{-1}\right)$; utilizar uma média mensal regional e utilizar o valor fixo de 2,0 $\mathrm{m} \mathrm{s}^{-1}$. De acordo com os autores, quando dados de $\mathrm{u}_{2}$ não estão disponíveis, os melhores procedimentos para o cálculo da $\mathrm{ET}_{\mathrm{o}} \mathrm{PM}-\mathrm{FAO}$ com dados mínimos consistem em utilizar a $\mathrm{u}_{2}$ média anual regional ou importar dados de estações próximas.

No Brasil, neste mesmo tipo de abordagem de estimativa da $\mathrm{ET}_{\mathrm{o}} \mathrm{PM}-\mathrm{FAO}$ apenas com dados de temperatura máxima e mínima do ar, Minuzzi et al. (2014) não recomendam tal procedimento para as condições climáticas de Santa Catarina, devendo-se recorrer a outros métodos de estimativa de $E T_{0}$ diária que necessitem apenas dessa variável. Elevados valores de RMSE foram encontrados por Alencar et al. (2015) em diferentes localidades do Estado de Minas Gerais e por Morais et al. (2015) no Submédio Vale do São Francisco, quando a $\mathrm{ET}_{\mathrm{o}} \mathrm{PM}-\mathrm{FAO}$ foi estimada com dados mínimos, com a radiação solar estimada a partir das temperaturas máxima e mínima do ar, umidade relativa com a temperatura mínima e $\mathrm{u}_{2}$ de $2 \mathrm{~m} \mathrm{~s}^{-1}$.

Diferentemente dos resultados reportados no presente trabalho, na Tunísia Jabloun e Sahli (2008) consideraram a u2 média anual para cada local em estudo, radiação solar computada a partir das temperaturas máxima e mínima do ar e a pressão parcial de vapor a partir da temperatura mínima do ar; de acordo com os 
resultados, especialmente para as localidades de Kairouan, Kef e Sidi-Bouzid reportaram estimativas precisas de $\mathrm{ET}_{0}$ PM-FAO com dados mínimos quando comparado com a $\mathrm{ET}_{0}$ PM-FAO com dados completos, com valores de RMSE e MBE variando de 0,411 a 0,80 $\mathrm{mm} \mathrm{dia}^{-1}$ e de $-0,103$ a $0,078 \mathrm{~mm} \mathrm{dia}^{-1}$, respectivamente.

No estudo de Wang et al. (2011) nas condições climáticas do Malaui, foram estimadas a radiação solar e umidade relativa do ar a partir das temperaturas máxima e mínima e utilizada a u2 fixa de $2,0 \mathrm{~m} \mathrm{~s}^{-1}$; pois de acordo com os resultados, em algumas localidades este procedimento foi adequado nas estimativas da $\mathrm{ET}_{0}$ PM-FAO, já em outras localidades as estimativas de $\mathrm{ET}_{0}$ foram imprecisas. De acordo com Córdova et al. (2015), ao utilizarem a média global de u2 de $2,0 \mathrm{~m} \mathrm{~s}^{-1}$, os autores verificaram bons resultados, sugerindo que os cálculos de $\mathrm{ET}_{\mathrm{o}}$ podem não ser muito sensíveis à uz em climas úmidos frios como nos Andes do Equador.

No Brasil, mais precisamente no Estado do Ceará, aplicando procedimento similar para estimativa da radiação solar global e umidade relativa do ar, Silva et al. (2010a) reportaram as melhores estimativas de $\mathrm{ET}_{0}$ PM-FAO quando comparado a $\mathrm{ET}_{0}$ PM-FAO com dados completos para as condições climáticas do município de Sobral, ao utilizarem os valores constantes de $\mathrm{u}_{2}$ na faixa de 1,0 a 3,0 $\mathrm{m} \mathrm{s}^{-1}$. Já na localidade de Campos Sales, Silva et al. (2010b) registraram as melhores estimativas de $\mathrm{ET}_{0}$ PM-FAO com dados mínimos, especialmente na faixa de $\mathrm{u}_{2}$ de 2,5 a $5,0 \mathrm{~m} \mathrm{~s}^{-1}$. Silva et al. (2013) registraram as melhores estimativas de $\mathrm{ET}_{0}$ PM-FAO com dados mínimos, em Crateús, com un de 1,5 a $3,0 \mathrm{~m} \mathrm{~s}^{-1}$; $\mathrm{u}_{2}$ de 2,5 a
4,5 $\mathrm{m} \mathrm{s}^{-1}$ em Quixeramobim e $\mathrm{u}_{2}$ de 1,5 e 2,0 $\mathrm{m} \mathrm{s}^{-1}$ em Tauá.

Estes resultados reforçam os resultados dos estudos de Rocha et al. (2011) na Bacia do Jaguaribe no Estado do Ceará e de Carvalho et al. (2013) em Lavras, no Estado de Minas Gerais. De acordo com estes autores, a abordagem com dados mínimos é uma alternativa viável para a estimativa da $\mathrm{ET}_{\mathrm{o}}$ onde os dados de entrada necessários para PM-FAO 56 não estão disponíveis, consequentemente estimativas precisas de $\mathrm{ET}_{0}$ associada ao coeficiente de cultivo da cultura, implicando em um manejo da irrigação mais eficiente e que segundo Silva et al. (2010a), pode evitar o desperdício de água e diminuir o custo com energia elétrica no momento da irrigação.

No desenvolvimento de um programa computacional para facilitar as estimativas de ETo PM-FAO 56 com dados limitados, Gocic e Trajkovic (2010) validaram o mesmo com dados de uma estação meteorológica de Davis na Califórnia, Estados Unidos, recomendando-se este tipo de abordagem para a estimativa da $\mathrm{ET}_{0} \mathrm{PM}$ FAO quando não há dados disponíveis. Nas condições climáticas do nordeste da Península Ibérica, também se recomenda esse mesmo tipo de abordagem para estimativa da $\mathrm{ET}_{0}$ com dados mínimos (LÓPEZ-MORENO et al., 2009).

Para o cenário de estimativas de $\mathrm{ET}_{0}$ PM-FAO 56 em que os dados em falta foram radiação solar global e velocidade do vento, ou seja, a umidade relativa do ar estava disponível, verificou-se um aumento no coeficiente de determinação, variando de 27,97\% quando foi utilizada a $\mathrm{u}_{2}$ constante de $1 \mathrm{~m} \mathrm{~s}^{-1}$ (Figura 2a) a 36,99\% ao se utilizar a $\mathrm{u}_{2}$ de $4 \mathrm{~m} \mathrm{~s}^{-1}$ (Figura 2d), consequentemente ocorreu uma redução nos erros. 
Silva et al.
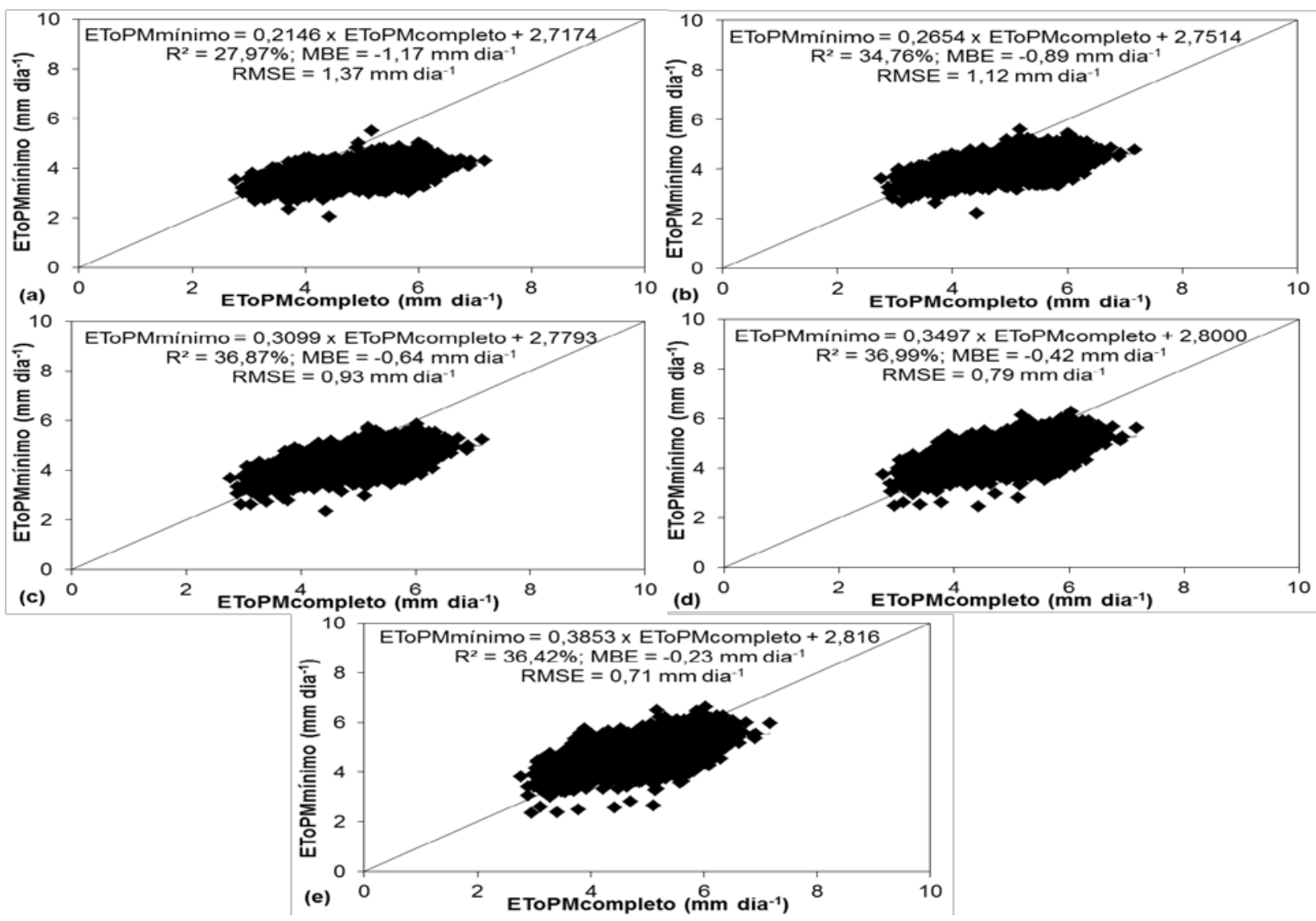

Figura 2. Comparação entre a $\mathrm{ET}_{0} \mathrm{PM}-\mathrm{FAO} 56$ com dados completos e $\mathrm{ET}_{0} \mathrm{PM-FAO} 56$ com dados mínimos (radiação solar global estimada a partir das temperaturas máxima e mínima do ar) e valores fixos de velocidade do vento de $1 \mathrm{~m} \mathrm{~s}^{-1}$ (a), $2 \mathrm{~m} \mathrm{~s}^{-1}$ (b), $3 \mathrm{~m} \mathrm{~s}^{-1}$ (c), $4 \mathrm{~m} \mathrm{~s}^{-1}$ (d) e $5 \mathrm{~m} \mathrm{~s}^{-1}$ (e).

Os valores de MBE e RMSE oscilaram de $-1,17$ a $-0,23 \mathrm{~mm}$ dia $^{-1}$ e de 1,37 a $0,71 \mathrm{~mm}$ $\operatorname{dia}^{-1}\left(\mathrm{u}_{2}\right.$ de 1 a $5 \mathrm{~m} \mathrm{~s}^{-1}$ ). Porém, com base nestes valores estatísticos encontrados para as condições climáticas de Aracaju, no Estado de Sergipe, mesmo quando a umidade relativa do ar estiver disponível não é recomendado estimar a $\mathrm{ET}_{0}$ PM-FAO, assumindo a estimativa da radiação solar global a partir das temperaturas máxima e mínima do ar. Isto mostra que a radiação solar é o parâmetro de maior impacto sobre as estimativas de $\mathrm{ET}_{\mathrm{o}}$ PM-FAO 56 com dados mínimos em locais litorâneos. O que pode ser comprovado no estudo de Carvalho et al. (2015) na região
Sudeste do Brasil, é que segundo este autor o cálculo da $\mathrm{ET}_{\mathrm{o}} \mathrm{PM}-\mathrm{FAO}$ com estimativa da radiação solar a partir das temperaturas máxima e mínima do ar não deve ser utilizado nas regiões litorâneas.

No cenário em que a radiação solar (obtida em função da insolação) estava disponível, confirmando o que foi dito anteriormente, ou seja, as estimativas de $\mathrm{ET}_{\mathrm{o}}$ PM-FAO com dados de umidade relativa do ar obtida a partir da temperatura mínima do ar e valores constantes de velocidade do vento foram mais precisas quando comparado com $\mathrm{ET}_{\mathrm{o}} \mathrm{PM}-\mathrm{FAO}$ com dados completos (Figura 3). 

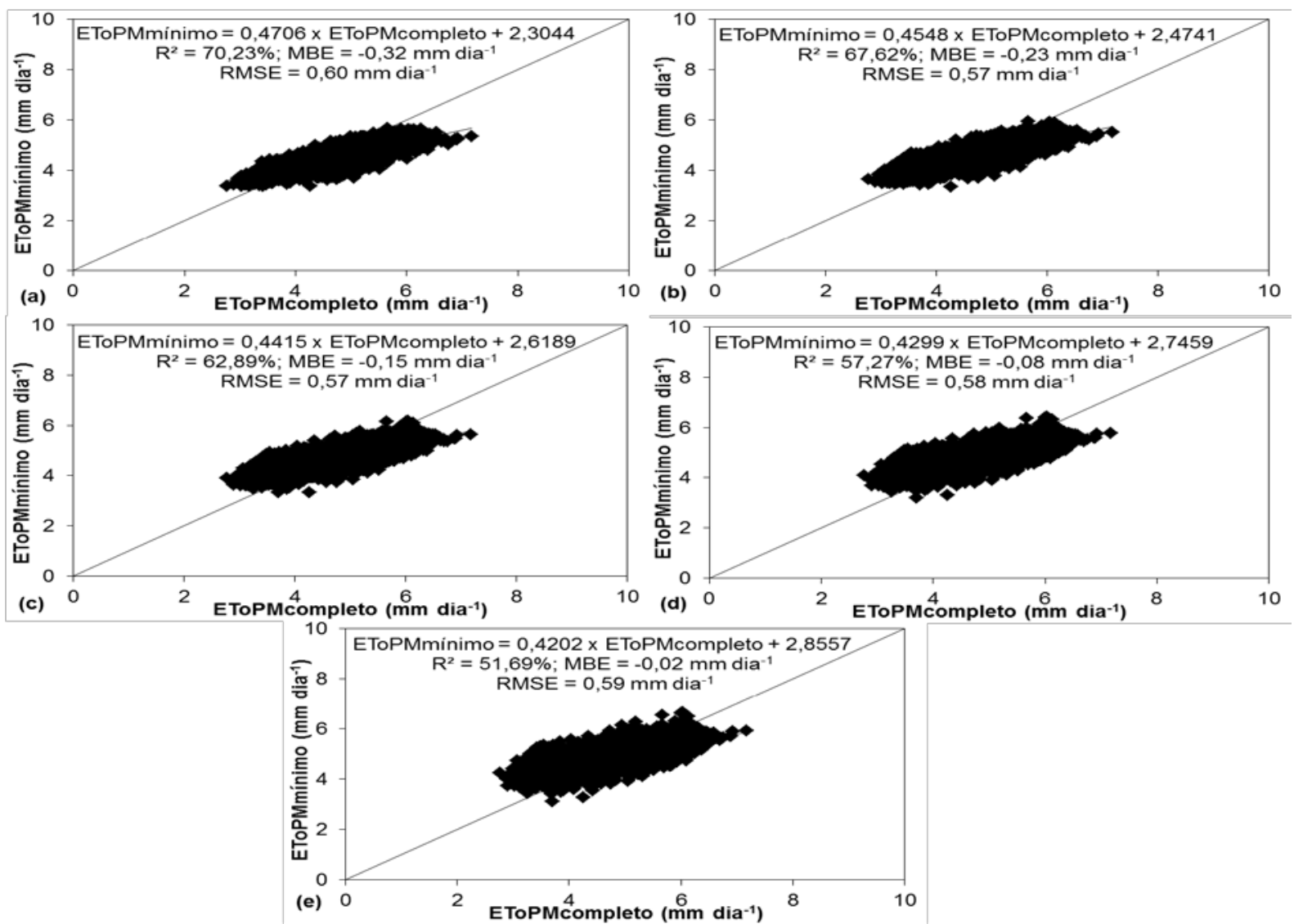

Figura 3. Comparação entre a $\mathrm{ET}_{0} \mathrm{PM}-\mathrm{FAO} 56$ com dados completos e $\mathrm{ET}_{0} \mathrm{PM}-\mathrm{FAO} 56$ com dados mínimos (umidade relativa do ar estimada a partir da temperatura mínima do ar) e valores fixos de velocidade do vento de $1 \mathrm{~m} \mathrm{~s}^{-1}$ (a), $2 \mathrm{~m} \mathrm{~s}^{-1}$ (b), $3 \mathrm{~m} \mathrm{~s}^{-1}$ (c), $4 \mathrm{~m} \mathrm{~s}^{-1}$ (d) e $5 \mathrm{~m} \mathrm{~s}^{-1}$ (e).

Estes resultados corroboram com os apresentados por Sentelhas et al. (2010), pois os autores concluíram que no sul de Ontário no Canadá, o método FAO PM pode ser usado para estimar a $\mathrm{ET}_{\mathrm{o}}$, razoavelmente, quando dados de velocidade do vento e/ou de umidade relativa não estiverem disponíveis. Ainda de acordo om os mesmos autores, quando utilizaram os procedimentos recomendados pelo boletim FAO 56 (ALLEN et al., 1998) para substituir dados em falta de $\mathrm{u}_{2}$ e $\mathrm{e}_{\mathrm{a}}$ (a partir da temperatura mínima do ar) ocorreu pouco impacto negativo sobre as estimativas de $\mathrm{ET}_{0}$, mas quando utilizaram o método recomendado para substituir dados em falta de radiação solar (a partir das temperaturas máxima e mínima do ar) não houve um bom desempenho.

Para as condições climáticas locais, com base no coeficiente de determinação, especialmente quando se utiliza os valores constantes de $\mathrm{u}_{2}$ na faixa de 1 a $3 \mathrm{~m} \mathrm{~s}^{-1}$, verifica-se melhor precisão nas estimativas de $\mathrm{ET}_{\mathrm{o}} \mathrm{PM}-\mathrm{FAO}$ 56; sendo os valores de RMSE da ordem de $0,60 \mathrm{~mm} \mathrm{dia}^{-1}$ (Figura 3a), 0,57 $\mathrm{mm} \mathrm{dia}^{-1}$ (Figura 3b) e 0,57 $\mathrm{mm} \mathrm{dia}^{-1}$ (Figura 3c). Em geral, os valores de MBE oscilaram de $-0,32 \mathrm{~mm} \mathrm{dia}^{-1}$ (u2 = $1 \mathrm{~m} \mathrm{~s}^{-1}$ ) (Figura 3a) a $-0,02 \mathrm{~mm} \mathrm{dia}^{-1}$ (u2 $=5 \mathrm{~m} \mathrm{~s}^{-1}$ ) (Figura 3e).

\section{CONCLUSÕES}

Para as condições climáticas da região em estudo, não se recomenda estimar a evapotranspiração de referência $\left(\mathrm{ET}_{0}\right)$ pelo método padrão Penman-Monteith FAO 56 quando se tem disponível apenas dados de temperaturas máxima e mínima do ar. Devendo-se recorrer a outros métodos alternativos que necessitem apenas das temperaturas máxima e mínima do ar em sua 
formulação, desde que, tais métodos sejam validados com o uso de lisímetros.

\section{REFERÊNCIAS BIBLIOGRÁFICAS}

ALLEN, R. G.; PEREIRA, L. S.; RAES, D.; SMITH, M. Crop evapotranspiration: guidelines for computing crop water requirements. Roma: FAO, 1998. 297p. (FAO Irrigation and Drainage Paper, 56).

ALENCAR, L. P.; SEDIYAMA, G. C.; MANTOVANI, E. C. Estimativa da evapotranspiração de referência (ETo padrão FAO), para Minas Gerais, na ausência de alguns dados climáticos. Engenharia Agrícola, Jaboticabal, v.35, n.1, p.39-50, 2015.

BENLI, B.; BRUGGEMAN, A.; OWEIS, T.; ÜSTÜN, H. Performance of PenmanMonteith FAO56 in a semiarid highland environment. Journal of Irrigation and Drainage Engineering, New York, v.136, n.11, p.757-765, 2010.

CARVALHO, D. F.; ROCHA, H. S.; BONOMO, R.; SOUZA, A. P. Estimativa da evapotranspiração de referência a partir de dados meteorológicos limitados. Pesquisa Agropecuária Brasileira, Brasília, v.50, n.1, p.1-11, 2015.

CARVALHO, L. G.; EVANGELISTA, A. W. P.; OLIVEIRA, K. M. G.; SILVA, B. M.; ALVES, M. C.; SÁ JÚNIOR, A.; MIRANDA, W. L. FAO Penman-Monteith equation for reference evapotranspiration from missing data. Idesia, Chile, v.31, n.3, p.39-47, 2013.

CÓRDOVA, M.; CARRILLO-ROJAS, G.; CRESPO, P.; WILCOX, B.; CÉLLERI, R. Evaluation of the Penman-Monteith (FAO 56 PM) method for calculating reference evapotranspiration using limited data. Mountain Research and Development, Washington, v.35, n.3, p.230-239, 2015.
GOCIC, M.; TRAJKOVIC, S. Software for estimating reference evapotranspiration using limited weather data. Computers and Electronics in Agriculture, New York, v.71, n.2, p.158-162, 2010.

JABLOUN, M.; SAHLI, A. Evaluation of FAO-56 methodology for estimating reference evapotranspiration using limited climatic data application to Tunisia. Agricultural Water Management, Amsterdam, v.95, n.6, p.707-715, 2008.

LÓPEZ-MORENO, J. I.; HESS, T. M.; WHITE, S. M. Estimation of reference evapotranspiration in a mountainous Mediterranean site using the PenmanMonteith equation with limited meteorological data. Pirineos, Jaca, v.164, p.7-31, 2009.

MAJIDI, M.; ALIZADEH, A.; VAZIFEDOUST , M.; FARID, A.; AHMADI, T. Analysis of the effect of missing weather data on estimating daily reference evapotranspiration under different climatic conditions. Water Resources Management, Amsterdam, v.29, p.21072124, 2015.

MARTINEZ, C. J.; THEPADIA, M. Estimating reference evapotranspiration with minimum data in Florida. Journal of Irrigation and Drainage Engineering, New York, v.136, n.7, p.494-501, 2010.

MINUZZI, R. B.; RIBEIRO, A. J.; SILVA, D. O.; KUNESKI, A. C. Estimativa da evapotranspiração de referência diária por Penman-Monteith FAO com dados de temperatura do ar para Santa Catarina. Irriga, Botucatu, v.19, n.3, p.548-558, 2014.

MOHAWESH, O. E. Evaluation of evapotranspiration models for estimating daily reference evapotranspiration in arid and semiarid environments. Plant Soil Environment, Prague, v.57, n.4, p.145-152, 2011. 

USANDO DADOS METEOROLÓGICOS LIMITADOS EM ARACAJU, SERGIPE

MORAIS, J. E. F.; SILVA, T. G. F.; SOUZA, L. S. B.; MOURA, M. S. B.; DINIZ, W. J. S.; SOUZA, C. A. A. Avaliação do método de Penman Monteith FAO 56 com dados faltosos e de métodos alternativos na estimativa da evapotranspiração de referência no Submédio Vale do São Francisco. Revista Brasileira de Geografia Física, Recife, v.8, n.6, p.16441660, 2015.

POPOVA, Z.; KERCHEVA, M.; PEREIRA, L. S. Validation of the FAO methodology for computing ETo with limited data. Application to south Bulgaria. Irrigation and Drainage, Slough, v.55, n.2, p.201-215, 2006.

ROCHA, E. J. T.; EVANGELISTA, S. R. M.; FUCK JÚNIOR, S. C. F.; GONDIM, R. S. Estimativa da Eto pelo modelo PenmanMonteith FAO com dados mínimos integrada a um Sistema de Informação Geográfica. Revista Ciência Agronômica, Fortaleza, v.42, n.1, p.75-83, 2011.

ROJAS, J. P.; SHEFFIELD, R. E. Evaluation of daily reference evapotranspiration methods as compared with the ASCE-EWRI PenmanMonteith equation using limited weather data in Northeast Louisiana. Journal of Irrigation and Drainage Engineering, New York, v.139, n.4, p.285-292, 2013.

SENTELHAS, P. C.; GILLESPIE, T. J.; SANTOS, E. A. Evaluation of FAO PenmanMonteith and alternative methods for estimating reference evapotranspiration with missing data in Southern Ontario, Canada. Agricultural Water Management, Amsterdam, v.97, n.5, p.635-644, 2010.

SILVA, I. N.; OLIVEIRA, J. B.; FONTES, L. O.; BRASIL, P. P.; SILVA, M. G. Estimativa da evapotranspiração de referência com dados mínimos para o cariri oeste cearense. Agropecuária Científica no Semiárido, Patos, v.6, n.3, p.42-48, 2010 b.

SILVA, M. G.; OLIVEIRA, J. B.; LÊDO, E. R. F.; ARAÚJO, E. M.; ARAÚJO, E. M. Estimativa da $\mathrm{ET}_{\mathrm{o}}$ pelos métodos Penman-
Monteith FAO 56 e Hargreaves-Samani a partir de dados de Tx e Tn para Sobral e Tauá no Ceará. Acta Tecnológica, Codó, v.5, n.2, p.52-68, 2010a.

SILVA, M. G.; ARRAES, F. D. D.; LEDO, E. R. F.; SANTOS, N. T.; SILVA FILHO, J. A. Avaliação da evapotranspiração de referência por Penman-Monteith usando dados climáticos mínimos no sertão do Ceará. Revista Agro@mbiente, Boa Vista, v.7, n.3, p.284293, 2013.

SILVA, M. G.; OLIVEIRA, I. S.; CARMO, F. F.; LEDO, E. R. F.; SILVA FILHO, J. A. Estimativa da evapotranspiração de referência pela equação de Hargreaves-Samani no Estado do Ceará, Brasil. Revista Brasileira de Engenharia de Biossistemas, Tupã, v.9, n.2, p.132-141, 2015.

TRAJKOVIC, S. Temperature-based approaches for estimating reference evapotranspiration. Journal of Irrigation and Drainage Engineering, New York, v.131, n.4, p.316-322, 2005.

TRAJKOVIC, S.; KOLAKOVIC, S. Estimating reference evapotranspiration using limited weather data. Journal of Irrigation and Drainage Engineering, New York, v.135, n.4, p.443-449, 2009.

TRAJKOVIC, S.; STOJNIC, V.; GOCIC, M. Minimum weather data requirements for estimating reference evapotranspiration. Architecture and Civil Engineering, Nis, v.9, n.2, p.335-345, 2011.

WANG, Y. M.; NAMAONA, W.; GLADDEN, L. A.; TRAORE, S.; DENG, L. T. Comparative study on estimating reference evapotranspiration under limited climate data condition in Malawi. International Journal of the Physical Sciences, v.6, n.9, p.2239-2248, 2011. 